\title{
FLIGHT PATH PLANNING FOR SMALL UAV LOW ALTITUDE FLIGHTS
}

\author{
Róbert SZABOLCSI \\ Óbuda University, Budapest, Hungary \\ szabolcsi.robert@bgk.uni-obuda.hu
}

\begin{abstract}
Recent days there are many projects of UAV urban applications, moreover, although in different habited area. Several concepts like urban drone taxi, flying boards, flying bikes, etc. are in phase of prototype system test and evaluation. As part of the modern robotization, UAVs are in the focus of attention of numerous scientists. UAVs, as special platforms are widely used by the Military in different scenarios and different flight missions. Capabilities of UAVS used in non-military applications (i.e. Police applications, disaster management applications, border control, firefighter applications, etc.) will serve well the idea of UAV 3D (Dirty-DullDangerous) application. The purpose of the author is to derive those low altitude UAV flight regimes when low altitude flight automation in necessary due to flight safety aspects, or, due to any existing regulations, and, finally, find and propose flight paths of the UAV better fitting given mission of the $U A V$.
\end{abstract}

KEYWORDS: automation, flight path planning, low altitude, altitude control, UAV

\section{Introduction}

Modern Unmanned Aerial Systems (UAS) are complex ones having air and ground units carrying out in lots of cases D3-missions. There are many new applications both in civilian and in military operations. Robotization is growing rapidly in UAV techniques, and numerous COTS UAV autopilots are at hand of the UAV users. Some autopilots are designed leaning on open source principle giving the floor for the UAV users to modify in a given meaning the autopilot software. Several UAV applications are planned to be executed at low altitude. In line with the modern trend of development of unmanned aerial systems, low altitude UAV flight and its automation are playing very important role (i.e. selfie drone applications). Regardless of the nature of the UAV applications, all kinds and all types of the UAVs must take-off for flight missions, and after successful flight missions they must be landed safely. Due to complexity of UAV take-off and landing in urban areas with proper landing zone real time selection, several UAVs are supplemented with autopilots, or, with automatic flight control systems to execute automated takeoff and automated landing of the UAV. The UAV low altitude safe flight capability is crucial for the Military. In sensitive military applications, approach and entering the operational theatre must be conducted at low heights to minimize its detection probability. Both for UAV civilian and governmental UAV use it is also important to conduct flights at extremely low heights to accomplish flight mission successfully. Mostly, the UAV reconnaissance flight 
missions are considered and developed in this paper.

\section{Related Works}

The excellent pioneer work of Rabinovich (1966) is introduced optimal flight path planning for cruising missiles in those early years of modern control engineering era. The missile low altitude terrain-following missions were investigated and optimal flight path was planned to maximize effectiveness of solution of the navigation problems. Aircraft low altitude flight is very important from many aspects of safety and security. Both open and closed loop automatic flight control systems design and analysis are in the focus of attention since many decades. In the textbook of Aslanyan (1984) the classical control engineering approach was used to design and analyze automatic flight control systems of the aircraft, helicopters and guided missiles. On the contrary, in the work of McLean (1990) the modern control engineering approach was used to design optimal automatic flight control systems. In 2010, Beseda-Portas, Torre and Cruz discussed a problem of the multiply UAV applications, and flight path planner was tested in real UAV flight tested in simulators. The UAV R\&D projects in line with the work of Eng (2011) dealt with UAV emergency landing automation. Low altitude UAV flights in urban area were discussed in the work of Castelli, Sharghi, Harper and Shah (2015). Small UAV low altitude flight paths are discussed and evaluated in the work of Vestegren (2016). NATO had issued a standard NATO STANAG 4586 (Ed3) for UAV Control System (UCS) to ensure UAS interoperability, incorporating those flight scenarios and UAV skills needed for safe UAV operations (2012).

\section{and Flight Regimes \\ 3. UAVs Low Altitude Applicatios}

The NATO STANAG 4586 (2012) defines the UAVs flight path control regimes as follows below:

- launch;

- waypoint (fly to predefined

waypoint(s));

- loiter;

- terrain avoidance;

- autoland engage;

- autoland wave-off,

- and following vehicle steering commands and flight control system regimes are available:

- angular speed (roll rate, pitch rate, yaw rate) control;

- attitude (angular) control;

- altitude control;

- speed (thrust) control;

- vertical speed control;

- heading control;

- loiter (latitude, longitude) control.

Easy to understand that UAV altitude is among those flight parameters being controlled and limited to ensure flight safety. Further, the terrain-following capabilities of the UAV will be examined leaning on system approach.

\section{UAV Altitude Control Systems}

Prior to any kind of closed loop automatic flight control system design, the UAV kinematics must be subjected to thorough analysis, focusing mostly on low altitude flight regimes.

\subsection{UAV Terrain-Following Mission}

The UAV terrain-following capability or, in other words, the UAV obstacle avoidance capabilities are important issues to improve flight safety. Obstacles via the UAV flight path might be natural (trees, hills etc.), and artificial (buildings, antennas, chimneys, electrical systems, towers, missile launch pads, etc.) ones. 
Regarding features of the obstacles, a safe UAV flight path can be designed. The typical maneuver available for this is the go-around type maneuver, if there is no extreme and aggressive climb request. If so, the fly-by maneuver is suggested to use, which means at constant height of the flight to change flight direction angle to avoid collision with obstacles on the flight path.
The terrain-following system is for the height control of the UAV flying at extremely low altitudes. This UAV ability provides minimum probability of the radar detection for the UAV, and minimum probability of the loss of the UAV hitting the ground. The terrain-following flight path vertical plane maneuver can be evaluated using Figure no. 1 (Aslanyan, 1984).

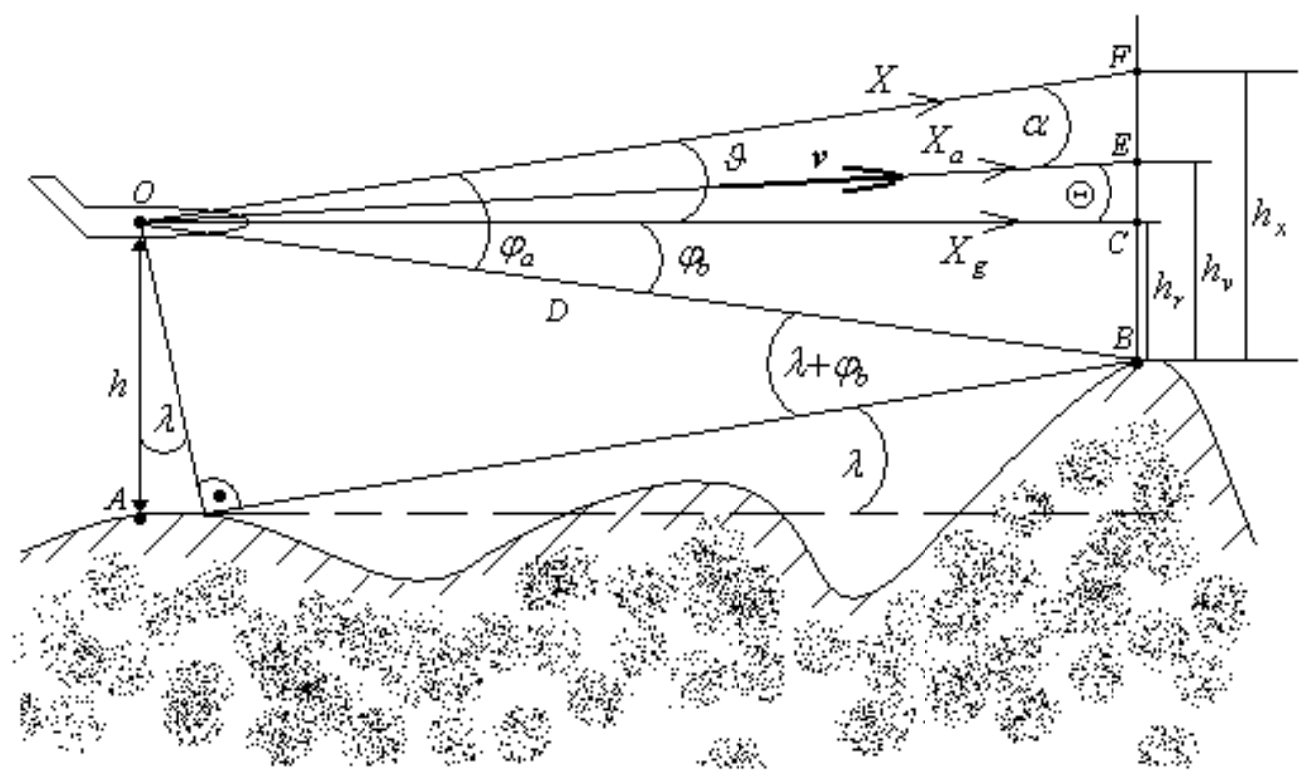

Figure no. 1: The UAV terrain-following geometry

In Figure no. 1: $\mathrm{O}$ - center of gravity of the UAV; A, B - surface peak points; D - forward distance between UAV and given point of the relief scanned by active sensors (i.e. radar beam, ultra sound, infrared signals, lasers etc.); $O X_{\mathrm{g}}-$ horizontal axis of the normal co-ordinate system of the UAV; $O X$ - longitudinal axis of the body-axis co-ordinate system of the $\mathrm{UAV} ; O X_{\mathrm{a}}-$ longitudinal axis of the aerodynamic co-ordinate system of the UAV; $\vartheta-$ pitch angle; $\alpha-$ AoA; $\Theta-$ flight path angle; $\lambda$ - relief slope angle; $\varphi_{a}=\varphi_{b}+\vartheta-$ angle between active signal beam and axis $O X$; and finally, $\varphi_{b}$ - angle between active signal beam and axis $O X_{\mathrm{g}}$.

Terrain-following of the UAV can be automated by keeping constant geographical height of the flight, $h$.
The automation of the UAV height stabilization as a standard procedure based upon distance (altitude) sensors like barometric devices, ultrasonic sensors, radio altimeters, laser gauges, or, infra range finders, which are very common gauges and techniques today in modern flight automation. It is easily can be seen that this principle can be applied only for regions having very few angles of the relief slope, otherwise ground hit probability is increasing, and one can crash UAV at the ground.

If there is any intensive variation of the relief slope this method can't be applied. To eliminate that drawback there are two options. The first is to maintain distance D constant, second one is to keep height of the flight in forward terrain point. 
4.1.1. Terrain-Following with Constant Forward Distance D

Using Figure no. 1 for triangle $\mathrm{OAB}$ following equation can be written:

$$
\frac{D}{\sin (\pi / 2-\lambda)}=\frac{h}{\sin \left(\varphi_{a}-\vartheta+\lambda\right)}
$$
as:

From eq (1) distance D can be found

$$
D=h \frac{\cos \lambda}{\sin \left(\varphi_{a}-\vartheta+\lambda\right)}
$$

Using small perturbation theory, in other words, Taylor-series expansion, equation (2) can be linearized, and it is represented in the following equation of small perturbations:

$$
\Delta D=K_{1} \Delta h+K_{2} \Delta\left(\varphi_{a}-\vartheta\right)+K_{3} \Delta \lambda
$$

where coefficients of equation (3) are as follows:

$$
\begin{gathered}
K_{1}=\left(\frac{\partial D}{\partial h}\right)_{O}=\left(\frac{D}{h}\right)_{O}, \\
K_{2}=\left(\frac{\partial D}{\partial \varphi_{b}}\right)_{O}=-\left[D \cdot \operatorname{ctg}\left(\varphi_{a}-\vartheta+\lambda\right)\right]_{O}, \\
K_{3}=\left(\frac{\partial D}{\partial \lambda}\right)_{O}=-\left[D \frac{\cos \left(\varphi_{a}-\vartheta\right)}{\sin \left(\varphi_{a}-\vartheta+\lambda\right) \cos \lambda}\right]_{O}
\end{gathered}
$$

From equation (5) and (6) it is easily can be derived that for small relief slopes $\lambda$, i.e. for near plane conditions: $K_{2} \cong K_{3}$.

Supposing that two active devices available on the UAV board for fast relief scanning, changes of the flight parameters are to be considered small ones, i.e. $\Delta \vartheta \cong 0$, and, $\Delta h \cong 0$. For this particular case, one can have following equations:

$$
\begin{aligned}
& D_{1}=h \frac{\cos \lambda}{\sin \left(\varphi_{a_{1}}-\vartheta+\lambda\right)}, \\
& D_{2}=h \frac{\cos \lambda}{\sin \left(\varphi_{a_{2}}-\vartheta+\lambda\right)},
\end{aligned}
$$

If to divide first equation of (7) by second one of (7) we can have following formula:

$$
\frac{D_{1}}{D_{2}}=\frac{\sin (\lambda-\vartheta) \cos \varphi_{a_{2}}+\cos (\lambda-\vartheta) \sin \varphi_{a_{2}}}{\sin (\lambda-\vartheta) \cos \varphi_{a_{1}}+\cos (\lambda-\vartheta) \sin \varphi_{a_{1}}},
$$

or, in other manner

$$
\begin{aligned}
& D_{1} \operatorname{tg}(\lambda-\vartheta) \cos \varphi_{a_{1}}+D_{1} \sin \varphi_{a_{1}}= \\
& \quad=D_{2} \operatorname{tg}(\lambda-\vartheta) \cos \varphi_{a_{2}}+D_{1} \sin \varphi_{a_{2}}
\end{aligned}
$$

The relief slope angle $\lambda$ can be derived from equation (9) as follows:

$$
\lambda=\vartheta+\operatorname{arctg}\left(\frac{D_{2} \sin \varphi_{a_{2}}-D_{1} \sin \varphi_{a_{1}}}{D_{1} \sin \varphi_{a_{1}}-D_{2} \sin \varphi_{a_{2}}}\right)
$$

Having distance D be measured, an automatic flight control system can be designed for stabilization of the reference distance, $D_{\text {ref }}$. Control law of the height control system providing constant distance, $\mathrm{D}$, can be derived as:

$$
D_{\text {ref }}-D=\frac{\Delta D}{\tau_{a} \mathrm{v}_{o}}
$$

where $\tau_{a}=m / \rho \mathrm{v} S$ is the aerodynamic time unit.

4.1.2. UAV Terrain-Following with Height Stabilization at the Forward Terrain Point

Height control in the pre-defined point method can be explained using Figure no. 1. Let us consider height of the flight $h_{\mathrm{v}}$ at point $B$ to be controlled. For the triangle $O B E$ one can write that:

$$
\frac{h_{\mathrm{v}}}{\sin \left(\varphi_{a}-\alpha\right)}=\frac{D}{\sin (\pi / 2-\Theta)}
$$

Equation (12) can be rewritten using formula (2) for distance D in the following way:

$$
h_{\mathrm{v}}=h \frac{\sin \left(\varphi_{a}-\alpha\right)}{\sin \left(\varphi_{a}-\vartheta+\lambda\right)} \frac{\cos \lambda}{\cos \Theta}
$$

It is known that $\alpha=\vartheta-\Theta$. Than equation (13) can be rearranged to have: 


$$
h_{\mathrm{v}}=h \frac{\sin \left(\varphi_{a}-\vartheta+\Theta\right)}{\sin \left(\varphi_{a}-\vartheta+\lambda\right)} \frac{\cos \lambda}{\cos \Theta}
$$

Using small perturbation theory nonlinear equation of (14) can be linearized, and represented in the following equation of small perturbations:

$$
\begin{aligned}
\Delta h_{v}= & K_{1 v} \Delta h+K_{2 v} \Delta\left(\varphi_{a}-\vartheta\right)+ \\
& +K_{3 v} \Delta \lambda+K_{4 v} \Delta \Theta
\end{aligned}
$$

where coefficients of equation (15) are as follows below:

$$
\begin{gathered}
K_{1 \mathrm{v}}=\left(\frac{\partial h_{\mathrm{v}}}{\partial h}\right)_{O}=\left(\frac{h_{\mathrm{v}}}{h}\right)_{O} \\
K_{2 \mathrm{v}}=\left(\frac{\partial h_{\mathrm{v}}}{\partial \varphi_{b}}\right)_{O}=\left[h_{\mathrm{v}} \frac{\sin (\lambda-\Theta)}{\sin \left(\varphi_{a}-\vartheta+\lambda\right) \sin \left(\varphi_{a}-\vartheta+\Theta\right)}\right]_{O} \\
K_{3 \mathrm{v}}=\left(\frac{\partial h_{\mathrm{v}}}{\partial \lambda}\right)_{O}=-\left[h_{\mathrm{v}} \frac{\cos (\lambda-\vartheta)}{\cos \lambda \cdot \sin \left(\varphi_{a}-\vartheta+\lambda\right)}\right]_{O} \\
K_{4 \mathrm{v}}=\left(\frac{\partial h_{\mathrm{v}}}{\partial \Theta}\right)_{O}=\left[h_{\mathrm{v}} \frac{\cos \left(\varphi_{a}-\vartheta\right)}{\cos \Theta \cdot \sin \left(\varphi_{a}-\vartheta+\Theta\right)}\right]_{O}
\end{gathered}
$$

An automatic UAV flight control system designed for stabilization of the reference height $h_{\mathrm{v}}$ at point $\mathrm{B}$, can be synthesized using following formula:

$$
h_{\mathrm{v} r e f}-h_{\mathrm{v}}=\frac{\Delta h_{\mathrm{v}}}{\tau_{a} \mathrm{v}_{o}}
$$

where $\tau_{a}=m / \rho \mathrm{v} S$ represents aerodynamic time unit.

Linearized equation of (15) contains term of the change of the flight path angle of $\Delta \Theta$, which provides high level flight safety during UAV low altitude flights. For small angles of relief slope of $\lambda$, and flight path angle of $\Theta$, equation (15) can be rewritten as follows

$$
\Delta h_{\mathrm{v}}=K_{1 \mathrm{v}} \Delta h+K_{2 \mathrm{v}} \Delta\left(\varphi_{a}-\vartheta\right)+K_{3 \mathrm{v}} \Delta(\lambda-\Theta)
$$

Using equation (21) it is evident that for derivation of the control law to be applied for height control of the UAV with given angle of $\varphi_{a}$, necessary to measure geographical height of the UAV, h, the pitch angle $\vartheta$, the flight path angle $\Theta$, and finally, the slope of the relief, $\lambda$. From Figure no. 1 it is evident that also the reference height $h_{\mathrm{r}}$ at point $\mathrm{B}$ can be stabilized to avoid direct flight into terrain: using Figure no. 1. for triangle $\mathrm{OBC}$ following equation can be derived:

$$
h_{\mathrm{r}}=D \cdot \sin \left(\varphi_{a}-\vartheta\right)
$$

Substituting equation (2) into (22) yields to:

$$
h_{\mathrm{r}}=h \frac{\cos \lambda \cdot \sin \left(\varphi_{a}-\vartheta\right)}{\sin \left(\varphi_{a}-\vartheta+\lambda\right)}
$$

Using small perturbation theory nonlinear equation of (23) can be linearized, and one can represent it following way:

$$
\Delta h_{\mathrm{r}}=K_{1 \mathrm{r}} \Delta h+K_{2 \mathrm{r}} \Delta\left(\varphi_{a}-\vartheta\right)+K_{3 \mathrm{r}} \Delta \lambda
$$

where coefficients of equation (24) are as follows below:

$$
\begin{array}{r}
K_{1 \mathrm{r}}=\left(\frac{\partial h_{\mathrm{r}}}{\partial h}\right)_{O}=\left(\frac{h_{\mathrm{r}}}{h}\right)_{O} \\
K_{2 \mathrm{r}}=\left(\frac{\partial h_{\mathrm{r}}}{\partial \varphi_{b}}\right)_{O}=\left[h_{\mathrm{r}} \frac{\sin \lambda}{\sin \left(\varphi_{a}-\vartheta\right) \sin \left(\varphi_{a}-\vartheta+\lambda\right)}\right]_{O}
\end{array}
$$

$$
K_{3 \mathrm{v}}=\left(\frac{\partial h_{\mathrm{v}}}{\partial \lambda}\right)_{O}=-\left[h_{\mathrm{v}} \frac{\cos \left(\varphi_{a}-\vartheta\right)}{\cos \lambda \cdot \sin \left(\varphi_{a}-\vartheta+\lambda\right)}\right]_{O}
$$

From Figure no. 1 it can be seen that also the reference height $h_{\mathrm{x}}$ at point $\mathrm{B}$ can be stabilized to avoid: using Figure no. 1 for 
triangle OBF following equation can be derived:

$$
\frac{h_{\mathrm{x}}}{\sin \varphi_{a}}=\frac{D}{\sin (\pi / 2-\vartheta)}
$$

Substituting formula (2) into equation (28) results in

$$
h_{\mathrm{x}}=h \frac{\cos \lambda \cdot \sin \varphi_{a}}{\cos \vartheta \cdot \sin \left(\varphi_{a}-\vartheta+\lambda\right)}
$$

Nonlinear equation of (29) can be linearized using Taylor series expansion for small perturbations. Thus, we have:

$$
\Delta h_{\mathrm{x}}=K_{1 \mathrm{x}} \Delta h+K_{2 \mathrm{x}} \Delta \varphi_{a}+K_{3 \mathrm{x}} \Delta \lambda+K_{4 \mathrm{x}} \Delta \vartheta(30)
$$

where coefficients of equation (30) are as follows:

$$
\begin{gathered}
K_{1 \mathrm{x}}=\left(\frac{\partial h_{\mathrm{x}}}{\partial h}\right)_{O}=\left(\frac{h_{\mathrm{x}}}{h}\right)_{O} \\
K_{2 \mathrm{x}}=\left(\frac{\partial h_{\mathrm{v}}}{\partial \varphi_{a}}\right)_{O}=\left[h_{\mathrm{x}} \frac{\sin (\lambda-\vartheta)}{\sin \left(\varphi_{a}-\vartheta+\lambda\right) \sin \varphi_{a}}\right]_{O} \\
K_{3 \mathrm{x}}=\left(\frac{\partial h_{\mathrm{x}}}{\partial \lambda}\right)_{O}=-\left[h_{\mathrm{x}} \frac{\cos \left(\varphi_{a}-\vartheta\right)}{\cos \lambda \cdot \sin \left(\varphi_{a}-\vartheta+\lambda\right)}\right]_{O}
\end{gathered}
$$

$$
K_{4 \mathrm{v}}=\left(\frac{\partial h_{\mathrm{x}}}{\partial \vartheta}\right)_{O}=\left[h_{\mathrm{x}} \frac{\cos \left(\varphi_{a}+\lambda\right)}{\cos \vartheta \cdot \sin \left(\varphi_{a}-\vartheta+\lambda\right)}\right]_{O}
$$

\subsection{UAV Loiter/Approach and Automatic Landing Flight Path Planning}

There are several methods for descend and landing used in UAV landing systems. The largest interest is in two methods going to be discussed latter.

\subsubsection{UAV Descend and Landing} Using Automatic Flare System

The UAV loiter control system is for automatic control of descend of the aircraft to the height of the pre-landing maneuver. The loiter geometry and algorithm can be derived using Figure no. 2.

The loiter flight path is started at $x=x_{1}$, from any height of the flight (entry point). The descend of the UAV is achieved at height of the flight derived by linear formula of $H(x)=H_{L O}(1-k x)$, where $\mathrm{k}$ is the slope of the descend flight path, which is often used as descend path design parameter, and finally, $H_{L O}$ is the loiter entry height.

The flight path is ended at height $H_{L E}=10 m$, at $x=x_{2}$, and this height of the flight is kept to be constant for the prelanding maneuver in $x_{3} \leq x \leq x_{2}$. In normal flight regimes the slope of the descend flight path is $\varepsilon=10^{\circ}$. For loiter maneuver control algorithm must be synthesized with consideration of the vertical speed, and vertical acceleration considering their limits, if there are any. To have safe enough loiter flight phase it is evident that vertical speed and acceleration must be limited to their maximum values. During approach/loiter following requirements are defined: $\left|\mathrm{v}_{L O}\right| \leq 0,8 \mathrm{v}_{\text {MAX }}, \Psi=$ const.,$|\gamma|=0^{\circ}$. 


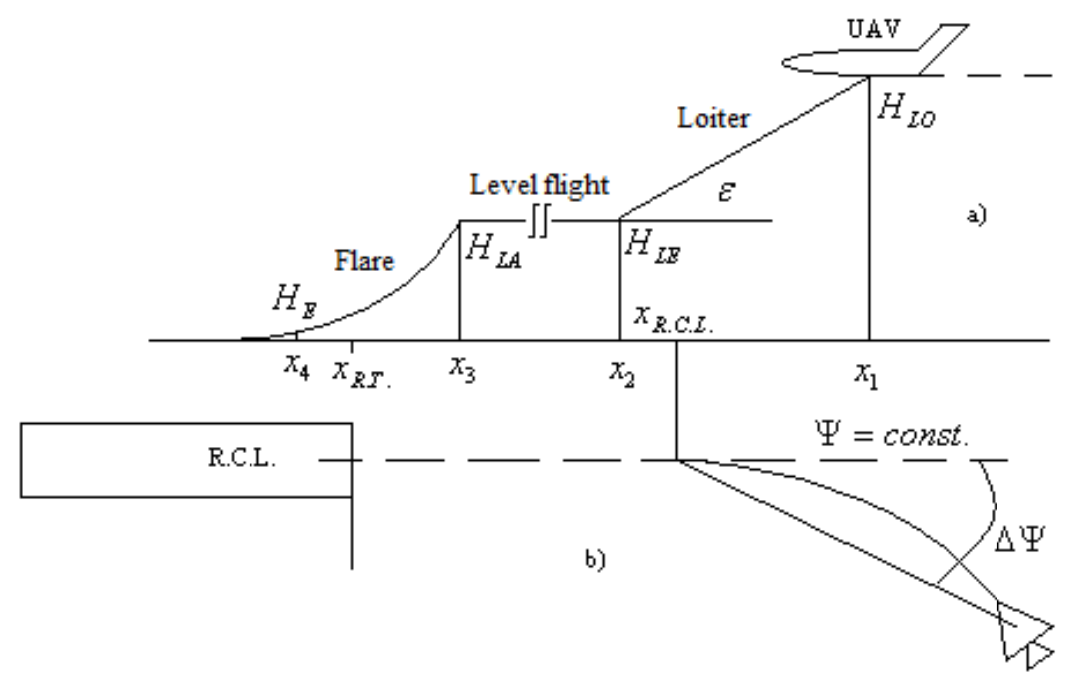

Figure no. 2: UAV system loiter and landing geometry

The landing system formally can be divided into two separated control channel, if the airspeed is constant. First control channel is the longitudinal, and the second is the lateral one. In the longitudinal control it is supposed that control parameter is the height of the flight varying by exponential function of $H(x)=H_{L A} \cdot e^{-\frac{x}{T}}$, where $T$ is the constant deriving slope of the exponential function. The UAV flare flight paths for different slopes are depicted in Figure no. 3. It is supposed that this flight phase ends at height $H_{E}=0,5 \mathrm{~m}$, at position of $x=x_{4}$. Depending on the sensor characteristics, used for altitude measurement, this altitude may vary. However, requirements for the sensor applied are to ensure the minimum of this distance measured to the runway.

In lateral meaning it is supposed that during approaching / loitering angular deflection measured from the runway center line (R.C.L.) $\Delta \Psi$ is minimized to its zero value. In other words, the control algorithm is $\Delta \Psi=0$ for the approaches both for the left and right side of the R.C.L.

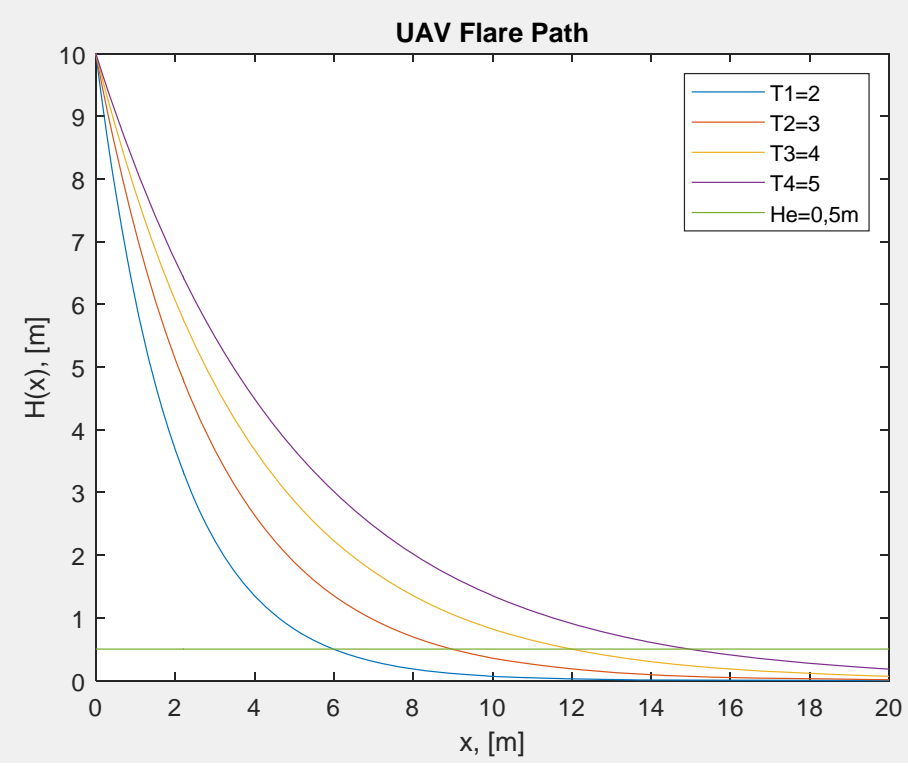

Figure no. 3: UAV system flare landing geometry (MATLAB - script is by the author) 
Regardless to emphasize that any profile of the UAV flare flight path must be selected with limitations on UAV vertical speed. In other words, if it required ensuring more smooth flight flare path, the flare entry point $x_{3}$ must be selected in accordance with these vertical speed limits.

\subsubsection{UAV Descend and Landing on} Linear Flight Paths

Difficulties related to generate exponential flight paths can be eliminated if to apply linear flight paths during landing (Vestegren, 2016). The simple flight path profile can be seen in Figure no. 4.

Prior to $x=x_{1}$ distance measured from the touchdown point, UAV is approaching in level flight at constant speed. At $x=x_{1}$, sharp at $H=H_{1}$, and in the region of coordinates of $x_{2} \leq x \leq x_{1}$, the UAV starts to descend on the linear height path derived by the following equation:

$$
H(x)=H_{1} \cdot\left(1-k_{1} x\right)
$$

At $x=x_{2}$, sharp at $H=H_{2}$, and in the region of coordinates of $x_{3} \leq x \leq x_{2}$ the UAV starts to descend on the linear height path derived by the following equation:

$$
H(x)=H_{2} \cdot\left(1-k_{2} x\right)
$$

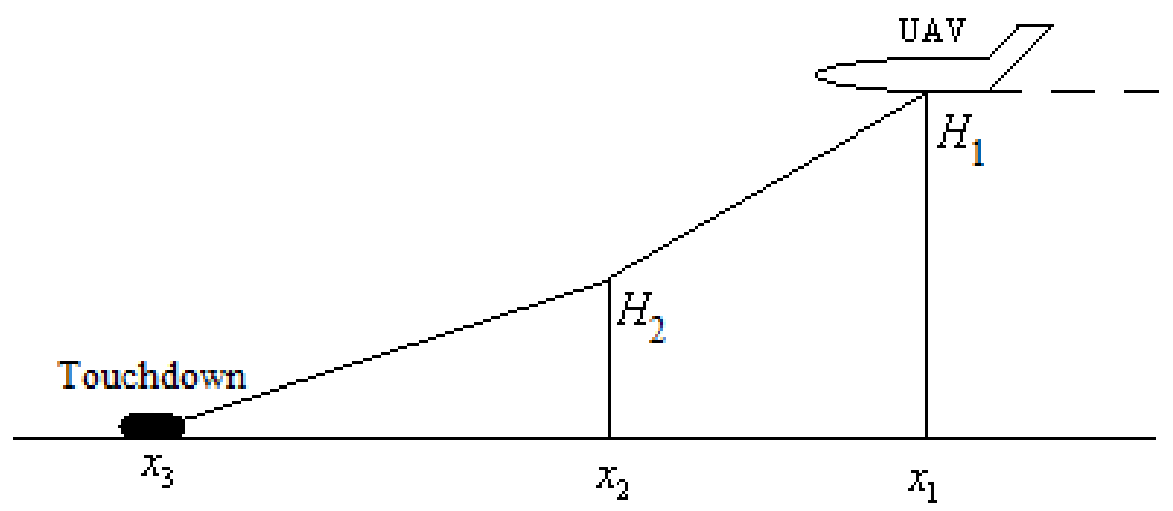

Figure no. 4: UAV system loiter and landing geometry

From Figure no. 4 it is easy to see that $k_{2}<k_{1}$. This will ensure the smooth touchdown on the runway. Selection of landing path geometry is strictly connected to, and also depends on UAV type. This system approach strongly depends on UAV flying characteristics and properties.

\section{Discussions}

The safe low altitude UAV flights can be achieved via automation. Automatic UAV flight control system will ensure not only the solution of the standard problem of height stabilization, but it can serve the purpose of avoidance of controlled flight into terrain.
Recent days the modern sensor system of the UAVs is able to sense altitude leaning on several methods. The terms incorporated into the control law derived by eq (15) are calculated based on sensor fusion. This control law allows to consider flight parameters, e.g. pitch angle, orientation of the speed vector (flight path angle), and relief slope angle. The UAV closed loop height control system provides high level flight safety.

The UAV closed loop automatic control system of the altitude control based upon control law of equation (24) misses information about airspeed vector orientation (flight path angle): Furthermore, there is a weak dependence on pitch angle, 
and as a consequence, this method can't provide high level flight safety, and its application must be avoided, or, one must take care about this bottleneck.

Altitude stabilization algorithm defined by equation (30) for control law is more accurate than that of the control law of (24), and incorporates signals of the pitch angle. However, it misses signal of flight path angle $\Theta$. It means that it can be applied with great attention paid to flight parameters and to variable relief conditions.

\section{Conclusions}

The paper deals with UAV low altitude flight path planning. There were discussed two main flight regimes. Namely, the UAV terrain-following missions and the UAV ground proximity alert system basics were in the focus of attention of the author, and also the UAV flight path planning during landing was discussed in this paper.
For terrain-following UAVs control laws were designed and introduced for altitude control automation, able to serve UAV closed loop control purposes.

Finally, the UAV landing procedure was investigated and two flight path profiles were introduced to be applied in vertical plane maneuvering. The linear height path of landing procedure was splitted into two different stages, with different path slopes. Depending on the need, or requirements set prior to path profile one can use more stages being linear ones with different negative slopes. The UAV landing system based upon its flare ensures more smooth touchdown minimizing load on UAV landing gears, which might be limited to minimize system ageing. Future work will target the low altitude flight path selection with consideration of both horizontal and vertical speeds, and accelerations, too.

\section{REFERENCES}

Aslanyan, A. E. (1984). Aircraft Automatic Flight Control Systems. Vol. I, Kiev Air Force Institute. Kiev, Ukraine: University Press.

Beseda-Portas, E., de la Torre, L., de la Cruz, J. M., \& Bonifacio, A.-T. (2010). Evolutionary Trajectory Planner for Multiply UAVs in Realistic Scenarios. IEEE Transactions on Robotics, Vol. 26, No. 4, 619-634.

Castelli, T., Sharghi, A., Harper, D., Tremeau, A., \& Shah, M. (2015). Autonomous navigation for low-altitude UAVs in urban areas. Engineering, Computer Science, Cornell University.

Eng, Pilar., C. S. (2011). Path Planning, Guidance and Control for a UAV Forced Landing. PhD thesis, Queensland University of Technology.

MATLAB R2019b, Control System Designer/Control System Toolbox 10.3. User's Guide. Natick, MA: The MathWorks Inc.

MATLAB R2019b, Parallel Computing Toolbox ${ }^{\mathrm{TM}}$ User's Guide. Natick, MA: The MathWorks Inc.

McLean, D. (1990). Automatic Flight Control Systems. Prentice Hall International Series in Systems and Control Engineering. New York: Prentice-Hall International Ltd.

NATO. (2012). STANAG 4586 - Standard Interfaces of UAV Control System (UCS) for NATO Interoperability. $3^{\text {rd }}$ Edition. NSA/123582012) 4586.

Rabinovich, B.I. (1966). Optimal Flight Path Design for Cruising Missiles. Moscow, Russia: Mashinostroenie Publishers.

Vestegren, M. (2016). Automatic Takeoff and Landing of Unmanned Fixed Wing Aircraft. A System Engineering Approach. Master of Science Thesis in Computer Science, Linköping University. 